\title{
An "Extra Dimension" in Electron Tomography: Automatic Parameter Determination for Next-generation Reconstruction Methods
}

\author{
Yi Jiang ${ }^{1}$, Nina Andrejevic ${ }^{2}$, Elliot Padgett ${ }^{2}$, David A. Muller ${ }^{2,3}$ \\ 1. Department of Physics, Cornell University, Ithaca, NY 14853 \\ 2. School of Applied and Engineering Physics, Cornell University, Ithaca, NY 14853 \\ 3. Kavli Institute at Cornell for Nanoscale Science, Ithaca, New York 14853
}

Electron tomography (ET) reconstructs a 3D model of materials using their 2D projections. Recently, there has been growing interest in developing optimization-based reconstruction techniques, often colloquially referred to as "compressed sensing electron tomography" [1], that obtain reconstructions by formulating and solving a specific optimization problem. Compared with traditional back projection, optimization-based methods are more flexible in using additional prior knowledge about the object to improve reconstruction quality with reduced sampling. However, extra parameters introduced in optimization problems often have significant impacts on images, yet are seldom explored in literature. Here, we demonstrate the use of a power spectrum analysis to facilitate robust automatic parameter selection for ET using the popular total variation (TV) minimization method.

As a proof of concept, we apply the so-called constrained-TV minimization method [2] to reconstruct a $2 \mathrm{D}$ test image resembling platinum nanoparticles on a porous carbon support. The optimization problem selects an image with minimum TV from a set of solutions that satisfy two constraints: 1) All pixels must be non-negative and 2) the Euclidean data residual of the reconstruction must be less than or equal to a data-tolerance parameter $\varepsilon$. Different values of $\varepsilon$ can lead to drastically different results (Figure 1).

Inspired by the work of Bian [3], we study how the power spectra of TV reconstructions behave as a function of the data-tolerance parameter. For each $\varepsilon$, we compute the power spectrum by averaging the reconstruction's Fourier intensity over the polar angle. Using a threshold, we further divide the spectrum into a signal region and a noise region (Figure 2a). As $\varepsilon$ decreases, the area under the signal spectrum (AUS) initially increases then reaches a plateau, whereas the area under the noise spectrum continues to increase (Figure 2b). Our simulations show that the data-tolerance parameter that minimizes the second derivative of $\operatorname{AUS}(\varepsilon)$ (at the end of the plateau) often leads to a reconstruction that is visually similar to the true object. The selection criterion is robust to a wide range of plausible thresholds in the power spectrum.

Figure 3 shows an application of the method to experimental STEM data of platinum nanoparticles on a carbon nanofiber. The TV reconstruction selected by the power spectrum criterion (Figure 3c) shows improvement over its back-projected counterpart in minimizing grainy texture in the carbon and also preserving the resolution of platinum particles. The constrained-TV minimization method and various parameter selection methods will be implemented in the open source tomography software tomviz (www.tomviz.org) [4]. 
[1] Z. Saghi et al, Nano letters, 11 (2011), p. 4666-4673.

[2] E. Sidky et al, Physics in medicine and biology 53 (2008), p.4777-4807.

[3] J. Bian et al, Physics in medicine and biology 59 (2014), p.2659-2685.

[4] R. Hovden et al, Microscopy and Microanalysis, 21 (2015), p.2407-2408.

[5] Y. Jiang acknowledges funding from DOE grant DE-SC0005827.

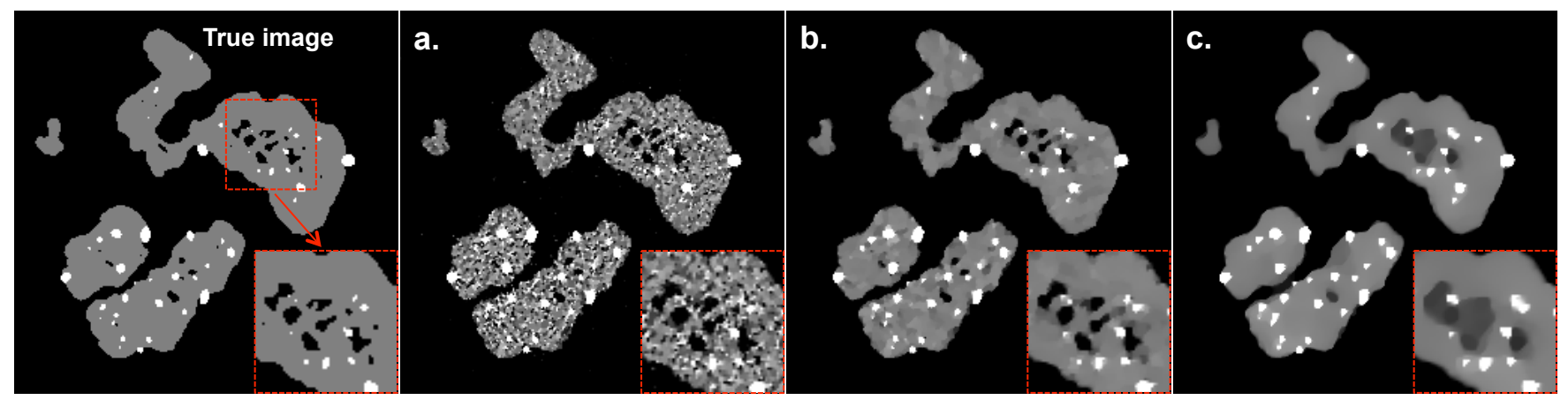

Figure 1. TV reconstructions of test image with different data-tolerance parameters. The simulated data is corrupted with Poisson noise and thus small $\varepsilon$ leads to grainy artifacts (a). Large $\varepsilon$ produces oversmoothed image (c) and reduces resolution. Reconstruction that is selected by the power spectrum analysis (b) is more similar to the true object.
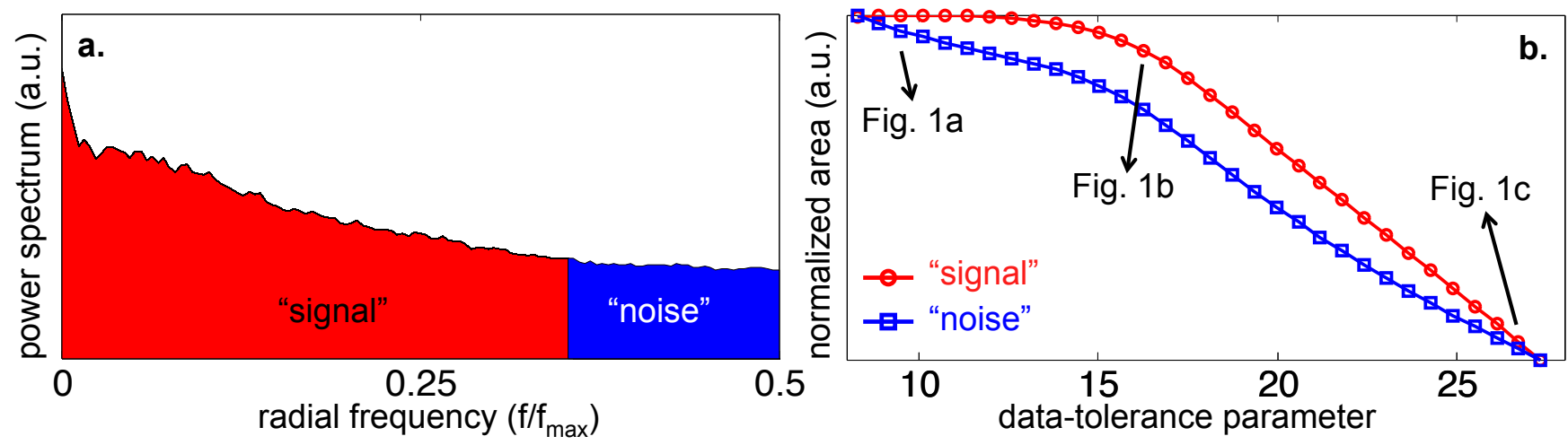

Figure 2. (a) Power spectrum (on a log scale) of a TV minimization reconstruction (Fig.1b). The spectrum is divided into two regions: signal and noise. (b) Areas under the signal (AUS) and noise (AUN) regions as functions of $\varepsilon$ (rescaled to fit the plot). As $\varepsilon$ decreases, AUS increases and reaches a plateau, indicating no further gain in information, while noise continues to increase with smaller $\varepsilon$.
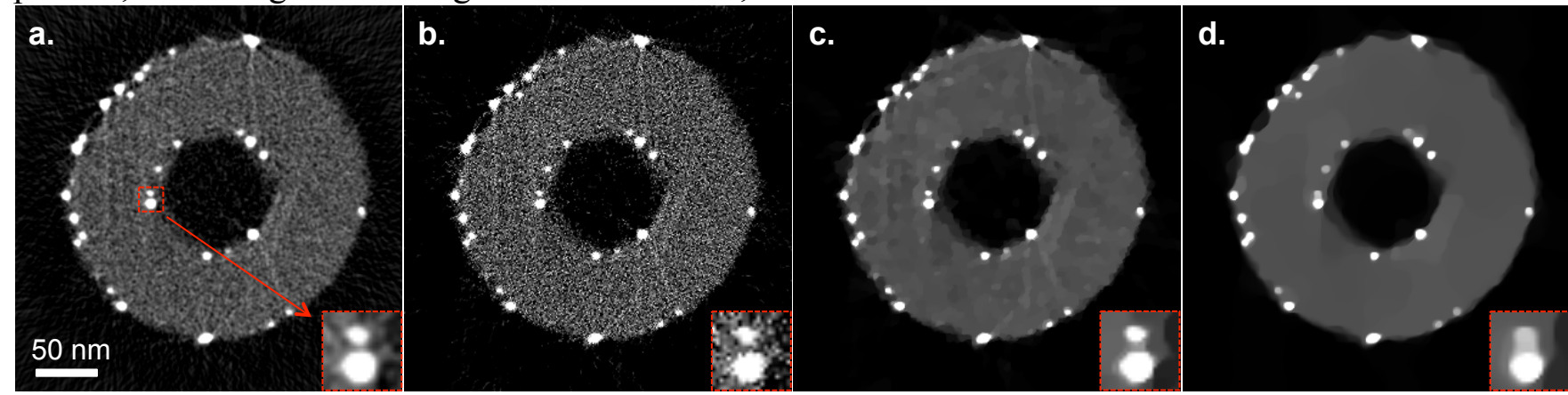

Figure 3. Reconstructions of platinum nanoparticles on carbon nanofiber using experimental data. A weighted back projection reconstruction is shown in (a) as a reference. The TV minimization reconstruction (c) selected by the power spectrum analysis is a reasonable compromise between noisy image (b) and over-smoothed image (d). 